\title{
Progressive Behavior Modification and Trending Pattern Analysis of Two Clinical Cases of Correlation Between T2d Patient Psychological Behavior and Physiological Characteristics (Using GH-Method: Math-Physical Medicine \& Mentality-Personality Modeling)
}

\author{
Gerald C Hsu
}

eclaireMD Foundation, USA

*Corresponding author
Gerald C Hsu, eclaireMD Foundation, USA

Submitted: 14 Apr 2020; Accepted: 20 Apr 2020; Published: 01 May 2020

\section{Introduction}

The author has contemplated a specific question:

Why do some type 2 diabetes (T2D) patients choose to face serious complications, including death, rather than change their lifestyle in order to control their diabetic conditions?

He discusses two different clinical cases linking patient's personality traits and psychological behavior with diabetes physiological characteristics. He named this approach as the Progressive Behavior Modification which is a part of the "Mentality-Personality Modeling".

Method

T2D patients have faced three major challenges:

1. Availability of accurate disease information with either physical evidence or quantitative proof, not just some general qualitative descriptions that may include false or commercial driven news over the internet (knowledge issue).

2. Awareness of disease status and overcome self-denial by moving to "psychological acceptance" in order to take effective action. The most difficult barrier to overcome is to have willpower, determination, and persistence on lifestyle change (psychology issues).

3. A non-invasive, effective, and ease of use technology-based tool to accurately predict outcomes and also guide patients (technology issue).

The author collected 17,046 glucose data for 241 days and generated 723 postprandial plasma glucose (PPG) waveforms. He decomposed them first and then further re-integrated them into three distinctive waveforms, i.e. Himalaya, Twin Peak, and Grand Canyon (see reference 1).

In summary, peak glucose values of these three patterns are determined by patient's knowledge and willpower for diet control on carbs/sugar intake amount; however, the dropping speeds of PPG value are dependent on the patient's knowledge and willpower for post-meal exercise. The Himalaya pattern is created via physical inactivity. Twin Peak is created via wrong exercise pattern (knowledge) and insufficient post-meal exercise (willpower on exercise), while Grand Canyon is created via correct exercise style (knowledge) and sufficient amount of exercise (willpower on exercise).

By analyzing the percentage of three distinctive patterns and comparing them against PPG time-series data of diet and exercise, each patient's personality traits and behavior psychology will be revealed clearly and the trend of the glucose movement can also be predicted, re-directed, and even possibly modified as well. The patients indeed can modify their behavior one step at a time, i.e. taking a little step on a smaller scale. This is what the author defined as a "progressive" behavior modification.

\section{Results}

Patient A started with his PPG at $280 \mathrm{mg} / \mathrm{dL}$ in 2010 and moving toward lower left direction (lower PPG) via acquiring correct knowledge and being persistent with his exercise regimen. $\mathrm{He}$ then identified the effectiveness of post-meal walking in 2015 but still fought with his craving for carbs/sugar. This combination effect of exercise knowledge/practice and carbs/sugar craving created a zig-zag path toward his ultimate end point at lower left corner of glucose wave. Finally, he reached to $116 \mathrm{mg} / \mathrm{dL}$ level after 2017. Case A demonstrated the patient's strong willpower/persistence and also struggling with control of his diet and exercise.

Patient B started using an AI-based tool to monitor and predict his PPG since 4/21/2018. He started from his glucose value at $230 \mathrm{mg} /$ dL. Initially, he followed the tool's scientific guidance on controlling his diet and exercising after each meal. He quickly brought his PPG level down to $149 \mathrm{mg} / \mathrm{dL}$; however, he was not persistent with his exercise routine and reduced his post-meal walking by one-third, resulting with his PPG level going up to $170 \mathrm{mg} / \mathrm{dL}$. Case B showed his psychological weakness on lacking exercise willpower [1-4]. 


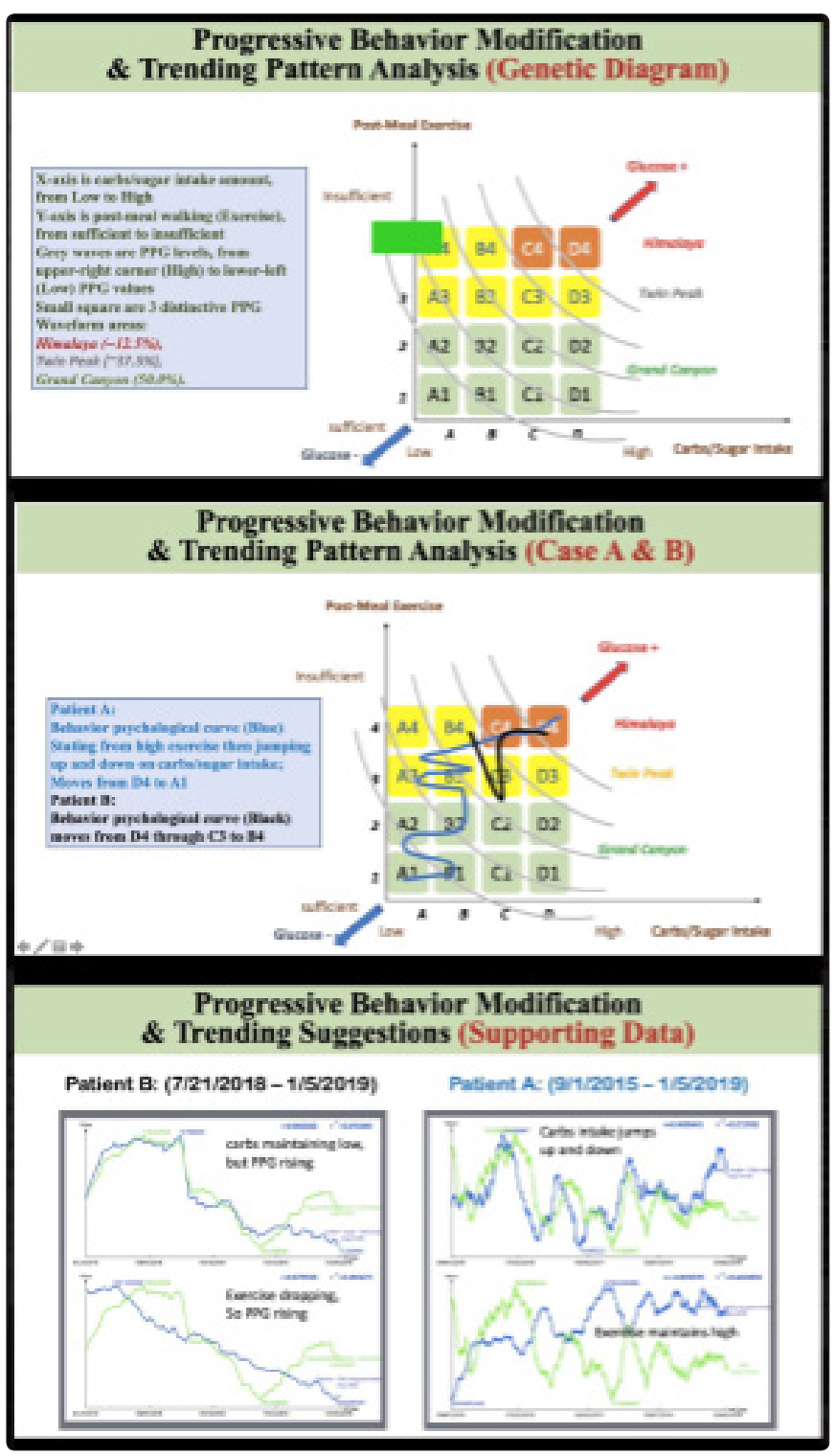

Figure: Comparison of glucose movement patterns due to different behavior psychology of two T2D patients

\section{Conclusion}

This paper is more of a forward-thinking article. The author believes that a big glucose data will be easily collected for T2D patients down the line with availability of non-invasive and easy-of-use glucose monitoring devices. Therefore, he is trying to lay the necessary groundwork for a future endeavor. Through analyzing those distinctive PPG waveforms, the personality traits and behavior psychological pattern of individual T2D patient can be revealed instantly and clearly. As a result, a more practical guidance of progressive behavior modification can be provided to T2D patients. There will be a lesser need to collect and analyze detailed data of food and exercise for this purpose.

\section{References}

1. Hsu Gerald C (2018) Using Math-Physical Medicine to Control T2D via Metabolism Monitoring and Glucose Predictions. Journal of Endocrinology and Diabetes 1: 1-6.

2. Hsu Gerald C (2018) Using Math-Physical Medicine to Analyze Metabolism and Improve Health Conditions. Video presented at the meeting of the 3rd International Conference on Endocrinology and Metabolic Syndrome Amsterdam, Netherlands.

3. Hsu Gerald C (2018) Using Signal Processing Techniques to Predict PPG for T2D. International Journal of Diabetes \& Metabolic Disorders 3: 1-3.

4. Hsu Gerald C (2018) Using Math-Physical Medicine and Artificial Intelligence Technology to Manage Lifestyle and Control Metabolic Conditions of T2D. International Journal of Diabetes \& Its Complications 2: 1-7.
Copyright: (2020 Gerald C Hsu. This is an open-access article distributed under the terms of the Creative Commons Attribution License, which permits unrestricted use, distribution, and reproduction in any medium, provided the original author and source are credited. 